\title{
Review of: "ECG pathology and its association with death in critically ill COVID-19 patients, a cohort study"
}

\author{
Giuliano Bolondi
}

Potential competing interests: The author(s) declared that no potential competing interests exist.

I thank the authors for their original study.

Unfortunately, it does not seem to profoundly impact everyday clinical practice. As commented, several studies have already shown the prognostic validity of biochemical data and of some ECG alterations in COVID-19 patients.

1. the decision to avoid multiple testing correction seems questionable and might provoke several pvalues to become non-significant.

2. to improve the quality of the work and make it suitable for pubblication, the authors could try to develop a prognostic score to predict mortality, length of stay or teh risk of intubation. Doing so, they can try to implement ECG and other clinical parameters (age, monitor saturation, quantity of oxygen needed, anamnesis, respiratory rate, ...) instead of biochemical data, making the score more economically sustainable and less stressful for the patients.

To date, in my personal opinion, I regret to say that the paper does not seem to be ready for publication. I would be happy to review a novel, more advanced version of the study.

Best regards. 